\title{
Platelets: the holy grail in cancer blood biomarker research?
}

\author{
Siamack Sabrkhany ${ }^{1} \cdot$ Marijke J. E. Kuijpers $^{2} \cdot$ Arjan W. Griffioen $^{3} \cdot$ Mirjam G. A. oude Egbrink $^{1}$
}

Published online: 20 October 2018

(c) The Author(s) 2018

\begin{abstract}
We would like to promote the fact that platelets are increasingly emerging as a rich source of potential biomarkers for cancer. Blood platelets contain vast amounts of bioactive proteins, such as growth factors, chemokines, and cytokines. These proteins are either synthesized by the megakaryocytes that produce the platelets or are sequestered by the circulating platelets from the blood, in which case these proteins may originate from the tumor. Recent studies in patients have demonstrated that the presence of cancer influences multiple platelet characteristics (e.g., platelet count, volume, activation status, proteins, and RNA content). Interestingly, these changes happened already in early stages of the disease before metastasis had occurred. Additionally, exploiting these platelet alterations enabled discrimination of patients with early-stage cancer from healthy sex- and age-matched individuals. Therefore, we challenge clinicians and researchers to look beyond traditional fluid sources such as plasma or serum, and to take platelets and their content into account as they may become the holy grail in cancer blood biomarker research.
\end{abstract}

Keywords Platelets $\cdot$ Cancer $\cdot$ Biomarkers

There is a need for blood biomarkers of cancer that can assist physicians in early detection and clinical decision-making during therapy. Thus far, most cancer blood biomarker studies are performed in plasma or serum, while the potential biomarker content of blood cells is overlooked. Especially platelets appear to be an important source of information in cancer blood biomarker research [1]. Platelets are the main transporters of growth factors, chemokines and cytokines in the circulation [2]. In addition, platelets have the capacity to sequester various types of bioactive molecules from their (micro)environment. This results in a distinct signature in their proteome and mRNA profile, which could reflect the presence of cancer $[3,4]$.

Mirjam G. A. oude Egbrink

m.oudeegbrink@maastrichtuniversity.nl

1 Department of Physiology, Cardiovascular Research Institute Maastricht, Maastricht University, P.O. Box 616, 6200 MD Maastricht, The Netherlands

2 Department of Biochemistry, Cardiovascular Research Institute Maastricht, Maastricht University, Maastricht, The Netherlands

3 Angiogenesis Laboratory, Department of Medical Oncology, Amsterdam University Medical Centre, Amsterdam, The Netherlands
Beside their role in hemostasis, platelets also play an important role in cancer progression, as they are able to secrete their rich content upon activation within the prothrombotic tumor microenvironment [2]. Although platelets contain both angiogenic and angiostatic proteins, their overall effect on cancer progression is stimulatory [2].

During the past years, platelets are emerging as a promising source of blood biomarkers for cancer. Platelets from patients with cancer have been shown to be phenotypically and functionally different compared to platelets from healthy controls [5-7]. Pretreatment thrombocytosis, i.e., increased platelet count, has long been recognized to be an independent predictor of poor prognosis in a variety of cancers such as lung, mesothelioma, breast, gynecologic, colorectal, gastric, and renal cancer [7]. In addition, recent studies also show that platelet content is affected by cancer presence. Peterson et al. [8] investigated several angiogenesis regulatory proteins in platelets of patients with colorectal cancer and compared this to the platelet content of age-matched healthy controls. They were able to demonstrate that the median VEGF, PDGF, and PF4 concentrations were higher in platelets of patients with colorectal cancer compared to controls. Using a combination of these values, they were able to discriminate patients with cancer from the control group with a high accuracy [8]. Interestingly, these protein 
concentrations were not affected in the plasma of patients, suggesting that changes in platelet content precede alterations in plasma concentrations of these angiogenesis regulatory proteins. In a prospective study, we recently identified changes in platelet count, volume, protein content, and activation status of patients with lung or head of pancreas cancer, when compared to platelets of healthy sex- and agematched controls [5]. Importantly, these changes occurred already in the early stages of cancer. While some of the changes were similar in both cancer groups, the precise pattern of changes showed to be cancer type specific. Furthermore, we were able to use a combination of changed platelet features to discriminate patients with lung or head of pancreas cancer from the healthy control group with a high accuracy. Additionally, in a different study, we determined the platelet proteome of patients with early-stage cancer. We demonstrated that the platelet proteome of patients with localized lung or head of pancreas cancer (stage I-II) is significantly different from that of healthy individuals of matched sex and age [4]. Moreover, the platelet proteome appeared to normalize after surgical resection of the malignant tumor. Hence, the platelet proteome may be mined for potential biomarkers of early-stage cancer [4].

Recently, Best et al. showed that the presence of cancer also alters the spliced RNA profile within platelets [3]. In this study, the platelet RNA of patients with non-small cell lung cancer was sequenced and compared to controls. Next, an algorithm was used to select a RNA biomarker panel which could be used to discriminate patients from controls. This platelet RNA panel was subsequently validated in an independent cohort, which resulted in a highly accurate discrimination of early- and late-stage cancer patients from healthy controls [3]. Overall, this study showed that changes in platelet RNA profile are a promising source for further cancer biomarker research.

While the studies mentioned above are encouraging, external validation of these results needs to be performed. Is it important to realize that platelets and their protein/RNA content are susceptible to manipulation and can easily be influenced during blood collection and processing $[3,5]$. Therefore, in future research, when measuring plateletstored biomarkers, it is imperative to use standardized blood collection, sampling and preparation in order to minimize ex vivo platelet activation. Also age and gender can significantly influence platelet characteristics and should be controlled for [3, 5]. Therefore, careful control group selection is crucial. It is imperative that patients with cancer are compared to a healthy sex- and age-matched control group. In addition, the cancer group may also be compared to patients who have a benign disease with similar clinical presentation as the cancer patient group. This could prove to be essential, as in actual clinical settings, cancer biomarkers are desired to distinguish patients with cancer from individuals with similar clinical symptoms that are at risk of having cancer. For example, to differentiate between patients with chronic obstructive pulmonary disease (COPD) and patients with COPD who have developed lung cancer.

In conclusion, during the past few years the assessment of platelet characteristics, content, as well as proteome or RNA profile has emerged as a new, promising and minimal invasive approach to detect (early-stage) cancer. Although further studies are needed to validate the above mentioned results, platelets may become the holy grail in cancer blood biomarker research.

Open Access This article is distributed under the terms of the Creative Commons Attribution 4.0 International License (http://creativeco mmons.org/licenses/by/4.0/), which permits unrestricted use, distribution, and reproduction in any medium, provided you give appropriate credit to the original author(s) and the source, provide a link to the Creative Commons license, and indicate if changes were made.

\section{References}

1. Best MG, Wesseling P, Wurdinger T (2018) Tumor-educated platelets as a noninvasive biomarker source for cancer detection and progression monitoring. Cancer Res 78(13):3407-3412

2. Sabrkhany S, Griffioen AW, oude Egbrink MG (2011) The role of blood platelets in tumor angiogenesis. Biochim Biophys Acta 1815(2):189-196

3. Best MG, Sol N, Sjors GJG, Vancura A, Muller M, Niemeijer ALN, et al (2017) Swarm intelligence-enhanced detection of nonsmall-cell lung cancer using tumor-educated platelets. Cancer Cell 32(2):238-252

4. Sabrkhany S, Kuijpers MJE, Knol JC, Olde Damink SWM, Dingemans AC, Verheul HM, Piersma SR, Pham TV, Griffioen AW, Oude Egbrink MGA, Jimenez CR (2018) Exploration of the platelet proteome in patients with early-stage cancer. J Proteomics 177:65-74

5. Sabrkhany S, Kuijpers MJE, van Kuijk SMJ, Sanders L, Pineda S, Olde Damink SWM, Dingemans AC, Griffioen AW, oude Egbrink MGA (2017) A combination of platelet features allows detection of early-stage cancer. Eur J Cancer 80:5-13

6. Sabrkhany S, Griffioen AW, Pineda S, Sanders L, Mattheij N, van Geffen JP, Aarts MJ, Heemskerk JW, Oude Egbrink MG, Kuijpers MJ (2016) Sunitinib uptake inhibits platelet function in cancer patients. Eur J Cancer 66:47-54

7. Lin RJ, Afshar-Kharghan V, Schafer AI (2014) Paraneoplastic thrombocytosis: the secrets of tumor self-promotion. Blood 124(2):184-187

8. Peterson JE, Zurakowski D, Italiano JE, Michel LV, Connors S, Oenick M, D’Amato RJ, Klement GL, Folkman J (2012) VEGF, PF4 and PDGF are elevated in platelets of colorectal cancer patients. Angiogenesis 15(2):265-273 